PREPARED FOR THE U.S. DEPARTMENT OF ENERGY, UNDER CONTRACT DE-AC02-76CH03073

PPPL-3778

PPPL-3778

UC-70

Lagrangian Formulation of Relativistic Particle Average Motion in a Laser Field of Arbitrary Intensity

by

I.Y. Dodin, N.J. Fisch, and G.M. Fraiman

February 2003

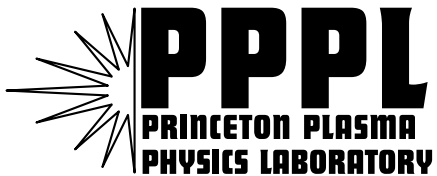

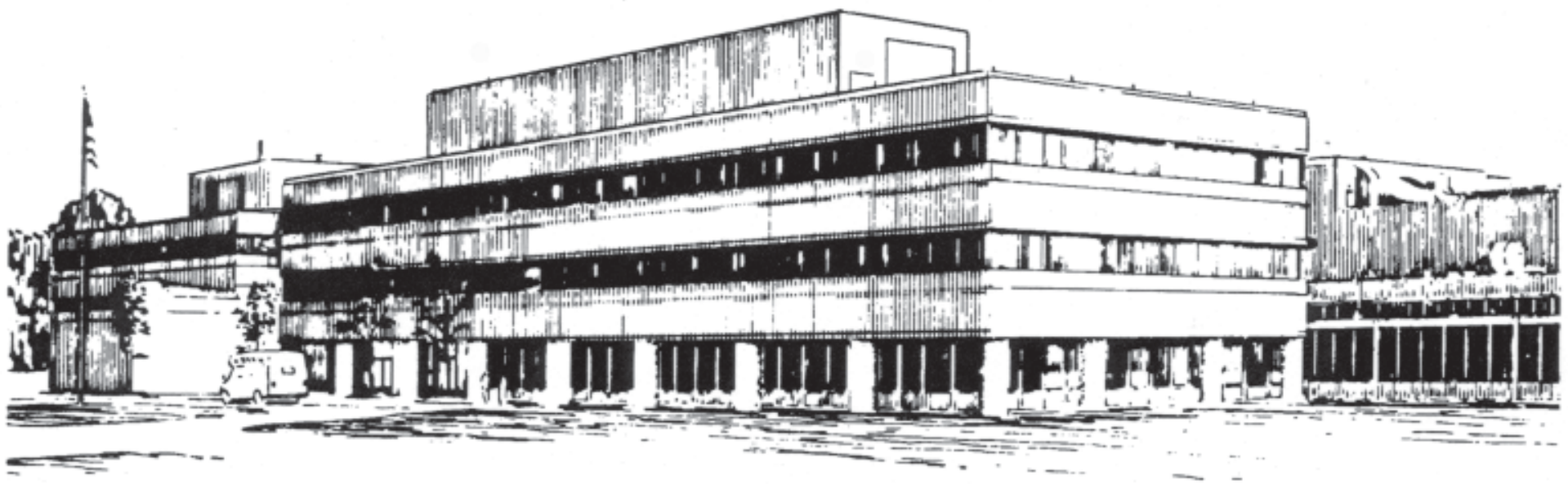

PRINCETON PLASMA PHYSICS LABORATORY PRINCETON UNIVERSITY, PRINCETON, NEW JERSEY 


\section{PPPL Reports Disclaimer}

This report was prepared as an account of work sponsored by an agency of the United States Government. Neither the United States Government nor any agency thereof, nor any of their employees, makes any warranty, express or implied, or assumes any legal liability or responsibility for the accuracy, completeness, or usefulness of any information, apparatus, product, or process disclosed, or represents that its use would not infringe privately owned rights. Reference herein to any specific commercial product, process, or service by trade name, trademark, manufacturer, or otherwise, does not necessarily constitute or imply its endorsement, recommendation, or favoring by the United States Government or any agency thereof. The views and opinions of authors expressed herein do not necessarily state or reflect those of the United States Government or any agency thereof.

\section{Availability}

This report is posted on the U.S. Department of Energy's Princeton Plasma Physics Laboratory Publications and Reports web site in Fiscal Year 2003. The home page for PPPL Reports and Publications is: http://www.pppl.gov/pub_report/

DOE and DOE Contractors can obtain copies of this report from:

U.S. Department of Energy

Office of Scientific and Technical Information

DOE Technical Information Services (DTIS)

P.O. Box 62

Oak Ridge, TN 37831

Telephone: (865) 576-8401

Fax: (865) 576-5728

Email: reports@adonis.osti.gov

This report is available to the general public from:

National Technical Information Service

U.S. Department of Commerce

5285 Port Royal Road

Springfield, VA 22161

Telephone: $1-800-553-6847$ or

(703) $605-6000$

Fax: (703) 321-8547

Internet: http://www.ntis.gov/ordering.htm 


\title{
Lagrangian formulation of relativistic particle average motion in a laser field of arbitrary intensity
}

\author{
I.Y. Dodin ${ }^{\dagger}$ and N.J. Fisch \\ ${ }^{\dagger}$ Princeton Plasma Physics Laboratory, Princeton, NJ 08543 \\ G.M. Fraiman \\ ${ }^{\ddagger}$ Institute of Applied Physics RAS, 46 Ulyanova Str., Nizhnii Novgorod, Russia 603600
}

\begin{abstract}
The Lagrangian and Hamiltonian functions describing average motion of a relativistic particle under the action of intensive high-frequency electromagnetic radiation are obtained. In weak, lowfrequency background fields, such a particle on average drifts with an effective, relativistically invariant mass, which depends on the intensity of the electromagnetic field.
\end{abstract}

PACS numbers: 52.35.Mw, 52.20.Dq, 52.27.Ny, 45.20.Jj

Recent advances in high-power laser technology have resulted in the development of laser systems capable of accelerating charged particles up to relativistic and even ultra-relativistic oscillatory velocities (see, e.g., Ref. $[1,2])$. This progress motivated the interest in theoretical models describing particles motion under the action of intense electromagnetic radiation. Particle motion is well-understood when the only forces present are those from the wave of uniform intensity [3]. However, in order to study the guiding center dynamics under the action of the forces arising from inhomogeneity of the laser field or from the presence of low-frequency background fields, additional analysis is needed.

The dynamics of a particle moving in a high-frequency field is often described in terms of so-called ponderomotive approach. In nonrelativistic ponderomotive description, the effect of high-frequency electromagnetic forces on a particle is replaced by interaction with an average potential, linear on the intensity of laser radiation $[4,5]$. When ultra-intense lasers are employed, the conventional ponderomotive description needs to be generalized to relativistic motion. Contrary to the degenerate case of a circularly polarized field, in which high-frequency variations of the relativistic mass can be neglected $[6,7]$, the problem of particle motion in the case of a linear or elliptic polarization represents a certain challenge, but still can be studied analytically, as shown below.

In order to describe the average particle dynamics in such fields, a number of analytical studies have been performed, which resulted in the development of the guiding center effective mass concept [8-12]. Namely, under different approximations, it was shown that the guiding center of an oscillating particle drifts in nonuniform laser field as a particle with an effective mass, which depends on the intensity of the electromagnetic field. The Hamiltonian treatment of the relativistic particle drift under the action of an intense laser radiation has been proposed in Ref. [13], though the problem of interaction with lowfrequency background fields has not been studied. The first steps towards including such interaction in the picture of particle drift motion in intense laser field were made in Ref. [9]. However, only large-scale (compared to the amplitude of oscillations) background fields were taken into consideration, the drift motion equations were generalized to the case of relativistic drifts without justification, and an incorrect expression was given for the relativistic ponderomotive potential.

These shortcomings are overcome in the present paper, which major emphasis is twofold. First, we propose a general, fully relativistic Lagrangian formulation of ponderomotive description of particle motion under the action of a monochromatic laser wave. The proposed approach is more systematic and simple in comparison with those discussed previously, and naturally allows to include particle interaction with weak background forces, additional to those from the laser field. The discussion of the guiding center motion in background fields constitutes the second emphasis of the paper. We show the effective mass concept to be applicable to ponderomotive description of relativistic drift motion in low-frequency background fields, including even ones of small spatial scale compared to the amplitude of particle oscillations. The latter application, for example, can be especially useful for studying the electron-ion scattering process in the presence of intense laser radiation, when oscillating electrons scatter on small-scale ion Coulomb potential [14$16]$.

To proceed, consider particle motion under the action of a plane electromagnetic (laser) wave propagating in vacuum with vector potential given by

$$
\boldsymbol{A}(\boldsymbol{r}, t)=\left(m c^{2} / e\right) \boldsymbol{a}(\eta)
$$

where $\eta=\omega t-\boldsymbol{k} \cdot \boldsymbol{r}$ stands for the phase of the wave, $\omega$ is the wave frequency, and $\boldsymbol{k}=\boldsymbol{z}^{0} \omega / c$ represents the wavevector. The polarization of the wave will be assumed fixed though arbitrary. The magnitude of $\boldsymbol{a}$, $a=e E / m c \omega$ (where $E$ is the laser electric field), can be understood as the ratio of the momentum imparted by the wave field in a single oscillation to $m c$, meaning that relativistic effects become important at $a \gtrsim 1$. (For the wavelength of the laser radiation equal to $\tilde{1} \mu \mathrm{m}$, the intensity corresponding to $a \sim 1$ for electrons is about $10^{18} \mathrm{~W} / \mathrm{cm}^{2}$.)

In a certain, unique, frame of reference, in such a 
field the particle undergoes stationary oscillatory "figureeight" motion in a linearly polarized wave or circular motion in a wave with circular polarization [3]. Averaging over the oscillations, one comes to the concept of the guiding center motion, which we study below. First, let us consider the variational principle that states the minimum value of the action

$$
S=\int_{t_{1}}^{t_{2}} L d t
$$

where $L$ stands for the Lagrangian function of the particle motion, to be realized on the true trajectory. On time scales $t_{2}-t_{1}$ large compared to the oscillation period, the major contribution to the action $S$ (linear on $t_{2}-t_{1}$ ) is provided by the time-averaged part of the Lagrangian, $\langle L\rangle$, while the contribution of the oscillatory Lagrangian into the integral (2) remains small. Thus, the action $S$ is approximately given by $S=\int_{t_{1}}^{t_{2}}\langle L\rangle d t$, from where it follows that $\langle L\rangle$ can be treated as the Lagrangian of the average, guiding center motion.

To obtain the form of the drift Lagrangian $L_{0} \equiv\langle L\rangle$, let us consider the latter in the frame of reference where the guiding center rests. In the new frame, the guiding center Lagrangian $\mathcal{L}_{0}$ can be nothing but a constant, which we put in the form

$$
\mathcal{L}_{0}=-m_{\mathrm{eff}} c^{2}
$$

in analogy with the Lagrangian of a true particle with zero velocity. The formally introduced quantity $m_{\mathrm{eff}}$ playing a role of a new, effective mass is yet to be defined. The action (2) is relativistically invariant and can be written as $S=\int_{\tau_{1}}^{\tau_{2}} \mathcal{L}_{0} d \tau$, where the time $d \tau$ represents the proper time of the guiding center. Since $d \tau$ is invariant by definition (and thus, so is the Lagrangian $\mathcal{L}_{0}$ ), the quantity $m_{\text {eff }}$ must also be relativistically invariant. Using

$$
d \tau=d t \sqrt{1-v_{0}^{2} / c^{2}}, \quad \mathcal{L}_{0} d \tau=L_{0} d t
$$

where $\boldsymbol{v}_{0}$ is the velocity of the guiding center in the original frame of reference, one gets the Lagrangian of the guiding center motion

$$
L_{0}=-m_{\mathrm{eff}} c^{2} \sqrt{1-v_{0}^{2} / c^{2}}
$$

which formally coincides with the Lagrangian of a relativistic particle with mass $m_{\mathrm{eff}}$ moving with velocity $\boldsymbol{v}_{0}$. Since the original frame was chosen arbitrarily, the above expression represents the general form of $L_{0}$, and what is left is to express $m_{\text {eff }}$ through the parameters of the laser field.

Such calculation is instructive to perform in a frame of reference where the particle has a nonzero average velocity $\boldsymbol{v}_{0}$. To proceed, consider the Lagrangian of particle true motion given by

$$
L=-m c^{2} \sqrt{1-v^{2} / c^{2}}+(e / c)(\boldsymbol{v} \cdot \boldsymbol{A}(\eta)),
$$

which is a known periodic function of the phase $\eta$ rather than time $t$. Thus, in order to average $L$ over time, one needs to derive a relation connecting time averaged and phase averaged quantities. For an arbitrary quantity $f$, its time and phase averaged values given by

$$
\langle f\rangle=\frac{1}{\Delta} \int_{t}^{t+\Delta} f d t^{\prime}, \quad \bar{f}=\frac{1}{2 \pi} \int_{\eta}^{\eta+2 \pi} f d \eta^{\prime},
$$

where the limits of integration over the phase correspond to the limits of integration over the time (i. e. $\eta=\eta(t)$ ), and the time interval $\Delta$ is defined as one on which the total phase change equals $2 \pi$ :

$$
\Delta=\int_{\eta}^{\eta+2 \pi} \frac{d t}{d \eta} d \eta
$$

The time interval $\Delta$ coincides with the wave period $2 \pi / \omega$ only if particle motion is nonrelativistic, so that $d \eta / d t \approx$ $\omega$, but, generally, the phase time-derivative is given by

$$
\frac{d \eta}{d t}=\omega\left(1-\frac{v_{z}}{c}\right)=\omega\left(\frac{\gamma-p_{z} / m c}{\gamma}\right) .
$$

Here $\gamma=\left(1-v^{2} / c^{2}\right)^{-1 / 2}$ is the normalized relativistic energy $\left(\mathcal{E}=m \gamma c^{2}\right)$ and $\boldsymbol{p}=m \gamma \boldsymbol{v}$ it the particle kinetic momentum.

Because of the fact that the original Lagrangian depends on $\eta$ (that is, on the combination $z-c t$, rather than $z$ and $t$ separately), there exists an invariant of motion given by

$$
u \equiv \gamma-p_{z} / m c=\text { const }
$$

Substituting the above expressions into Eq. (7), one gets

$$
\langle f\rangle=\overline{\gamma f} / \bar{\gamma}
$$

Note that the obtained expression is valid only in case when the electromagnetic wave (1) is propagating in vacuum. If the refraction index of the medium differs from unit, Eqs. (9) and (10) need to be modified, and the relation between time- and phase-averaged quantities becomes more complicated [13].

By using Eqs. (10) and (11), one gets

$$
\bar{\gamma}=\sqrt{1+(\bar{p} / m c)^{2}+\overline{a^{2}}}, \quad \bar{\gamma}=\gamma_{0} \sqrt{1+\overline{a^{2}}}
$$

where $\gamma_{0}=\left(1-v_{0}^{2} / c^{2}\right)^{-1 / 2}$, and $\boldsymbol{v}_{0} \equiv\langle\boldsymbol{v}\rangle=\overline{\boldsymbol{p}} / m \bar{\gamma}$ is the drift velocity. Thus, the Lagrangian $L_{0}$ can be put in the form (5) with $m_{\mathrm{eff}}$ given by

$$
m_{\mathrm{eff}}=m \sqrt{1+e^{2} \overline{A^{2}} / m^{2} c^{4}} .
$$

The guiding-center Lagrangian (5) with the expression (13) for the effective mass was also obtained in Ref. [12] 
by a similar, though not a straightforward procedure. However, in the cited work, Eq. (13) was supposed to be valid only in the frame of reference where $\boldsymbol{v}_{0}=0$. In fact, as shown above, it remains applicable for arbitrary $\boldsymbol{v}_{0}$, and, more than that, the actual value of $m_{\text {eff }}$ must be relativistically invariant. To express the effective mass in the invariant form, let us notice that, in the laboratory frame where we chose the electric potential $\phi=0$ (see Eq. (6)), $\sqrt{A^{2}}$ coincides with the norm of the 4-vector potential $\sqrt{A_{\alpha} A^{\alpha}}, A^{\alpha}=(\phi, \boldsymbol{A})$. The latter is Lorentzinvariant [17], and remains such after being averaged over relativistically invariant phase $\eta$. Thus, the expression for $m_{\mathrm{eff}}$, invariant to relativistic transformations, can be put in the following form:

$$
m_{\mathrm{eff}}=m \sqrt{1+\frac{e^{2}}{m^{2} c^{4}} \overline{\left(A_{\alpha} A^{\alpha}\right)}} .
$$

The expression (14) for the effective mass was also given in Refs. [8-10] where the average particle motion was studied otherwise.

Reverting to the formula for the drift Lagrangian (5) with the effective mass given by (13), the canonical momentum of the guiding center motion $\boldsymbol{P}_{0}$ equals the phase-averaged kinetic momentum $\overline{\boldsymbol{p}}=m_{\mathrm{eff}} \gamma_{0} \boldsymbol{v}_{0}$, and thus the Hamiltonian function of the guiding center motion can be put in the form

$$
H_{0}=\sqrt{m_{\mathrm{eff}}^{2} c^{4}+P_{0}^{2} c^{2}},
$$

where $m_{\text {eff }}$ may smoothly depend on the guiding center location $\boldsymbol{R}_{0}$ and time $t$ if the wave envelope, the particle interacts with, is slightly nonuniform or time-dependent:

$$
l \gg r_{\sim}, \quad T \gg \Delta,
$$

where $l$ and $T$ are the spatial and the temporal scales of the wave envelope inhomogeneity.

The Hamiltonian function (15) was previously derived in Ref. [13] by performing a sequence of canonical transformations of the original motion equations. In the present paper, we showed how this complicated procedure can be avoided. In addition, the proposed approach allows easy generalization of the drift Lagrangian and Hamiltonian formalism on the case when the oscillating particles undergo weak acceleration by large-scale lowfrequency forces satisfying (16). Interaction with additional forces of such kind enters the expression for $L_{0}$ additively and, what is most important, can still be considered in the framework of the effective mass concept.

To show this, consider an oscillating relativistic particle interacting with a field governed by the 4 -vector potential $A_{\mathrm{bg}}^{\alpha}=\left(\phi_{\mathrm{bg}}, \boldsymbol{A}_{\mathrm{bg}}\right)$, where the subindex "bg" stands for a background field, additional to the one of the laser wave. Assume that the field is weak in the sense that

$$
e E_{\mathrm{bg}} / \gamma_{0} m_{\mathrm{eff}} c \ll \omega, \quad e B_{\mathrm{bg}} / \gamma_{0} m_{\mathrm{eff}} c \ll \omega,
$$

where $\boldsymbol{E}_{\mathrm{bg}}$ and $\boldsymbol{B}_{\mathrm{bg}}$ are the corresponding electric and magnetic fields. In this case, the background fields do not impact the oscillatory motion significantly. Thus, averaging of the kinetic term $m c^{2} / \gamma$ in the Lagrangian leads to the same expression as in Eq. (5) with $m_{\text {eff }}$ given by Eq. (14). In the zeroth-order approximation with respect to the small parameters (17), the average part of the Lagrangian corresponding to particle interaction with the background field can be expressed in terms of the quantity $A_{0}^{\alpha}=\left(\phi_{0}, \boldsymbol{A}_{0}\right)$ given by

$$
A_{0}^{\alpha}=\left\langle A_{\mathrm{bg}}^{\alpha}\left(\boldsymbol{R}_{0}+\boldsymbol{r}_{\sim}\right)\right\rangle
$$

The time-averaging procedure is invariant with respect to changing the drift frame of reference, i. e. does not alter the Lorentz transformation properties of the quantity being averaged. Thus, $A_{0}^{\alpha}$ represents a true 4 -vector and can be considered as a new, effective electromagnetic field. In terms of this field's potentials, the drift Lagrangian can be put in the following form

$$
L_{0}=-m_{\mathrm{eff}} c^{2} \sqrt{1-v_{0}^{2} / c^{2}}+(e / c)\left(\boldsymbol{v}_{0} \cdot \boldsymbol{A}_{0}\right)-e \phi_{0} .
$$

In certain applications, it is of interest to consider the interaction with background fields having spatial scale $l$ comparable or small compared to the amplitude of particle oscillations $r_{\sim}$. If the drift velocity is small, such that the drift displacement on a single period $v_{0} \Delta$ is small compared to $l$, the ponderomotive description still can be applied. However, in this case the difference between the time-averaged potential $A_{0}^{\alpha}$ and the true potential $A_{\mathrm{bg}}^{\alpha}$ taken at the location of the guiding center $\boldsymbol{R}_{0}$, is crucial. For example, this situation is realized in the problem of Coulomb scattering in intense laser fields when $r_{\sim}$ exceeds the radius of effective interaction - see, e. g., Refs. [14-16]. For the latter, the considered Lagrangian approach represents a unique tool for studying both ponderomotive and even stochastic behavior of particles being scattered. This problem, however, deserves detailed consideration and will be discussed in future works. The important thing we would like to emphasize about Eq. (18) in the present context is that the amplitude of the effective potentials for a relativistic particle is the same than for a nonrelativistic one, and the only difference is provided through the change in the oscillatory trajectory $\boldsymbol{r} \sim$ to be averaged over.

Since, in the case of relativistic drift, $\boldsymbol{r}_{\sim}$ depends on $\boldsymbol{v}_{0}$, the expression for canonical momentum $\boldsymbol{P}_{0}=$ $\partial L_{0} / \partial \boldsymbol{v}_{0}$ becomes complicated and so do the drift motion equations. However, in two special cases of interest, those can be simplified. In a large-scale background field satisfying the conditions (16), locally, $A_{\mathrm{bg}}^{\alpha}$ can be treated as a linear function of $\boldsymbol{r}$. Therefore, the velocity-dependent part averages out when calculating the potential $A_{0}^{\alpha}$, and one gets $A_{0}^{\alpha} \approx A_{\mathrm{bg}}^{\alpha}$. Thus, the drift canonical momentum equals $\boldsymbol{P}_{0}=m_{\mathrm{eff}} \gamma_{0} \boldsymbol{v}_{0}+(e / c) \boldsymbol{A}_{\mathrm{bg}}$, and the Hamiltonian function is given by

$$
H_{0}=\sqrt{m_{\mathrm{eff}}^{2} c^{4}+\left(\boldsymbol{P}_{0}-(e / c) \boldsymbol{A}_{\mathrm{bg}}\right)^{2} c^{2}}+e \phi_{\mathrm{bg}}
$$


where the potentials are assumed to be slow functions of $\boldsymbol{R}_{0}$ and $t$. The guiding center motion equations can be put in the covariant form

$$
\frac{d R_{0}^{\alpha}}{d \tau}=\frac{p_{0}^{\alpha}}{m_{\mathrm{eff}}}, \quad \frac{d p_{0}^{\alpha}}{d \tau}=\frac{e}{c} F_{\mathrm{bg}}^{\alpha \beta} U_{\beta}-c^{2} \frac{\partial m_{\mathrm{eff}}}{\partial R_{0}^{\alpha}}
$$

where $R_{0}^{\alpha}=\left(c t, \boldsymbol{R}_{0}\right)$ is the 4-coordinate of the guiding center, $p_{0}^{\alpha}=\left(\mathcal{E}_{0} / c, m_{\mathrm{eff}} \gamma_{0} \boldsymbol{v}_{0}\right)$ is the drift kinetic 4-momentum, $\mathcal{E}_{0}=m_{\mathrm{eff}} \gamma_{0} c^{2}$ is the energy of the guiding center motion, $F_{\mathrm{bg}}^{\alpha \beta}$ is the electromagnetic field tensor corresponding to the potential $A_{\mathrm{bg}}^{\alpha}$ [17], and $U^{\alpha}=$ $\gamma_{0}\left(c, \boldsymbol{v}_{0}\right)$ is the guiding center 4 -velocity. Without derivation, the above equations were also given in Ref. [9].

From Eq. (20), it follows that in low-frequency largescale background field, the guiding center of a relativistic particle moving under the action of intense laser radiation behaves as a particle with the effective mass $m_{\text {eff }}$ drifting in the same background field. For example (and in coincidence with the results obtained in Ref. [9]), in static magnetic field $\boldsymbol{B}_{\mathrm{bg}}$, the guiding center undergoes Larmor motion with cyclotron frequency given by $\omega_{B}=e B_{\mathrm{bg}} / \gamma_{0} m_{\mathrm{eff}} c$. Conventional expression for the drift velocity in nonuniform magnetic field [17] also readily applies to the average motion if the particle true mass is replaced with the one given by Eq. (14).

In addition to the case of large-scale background fields, the guiding center motion equations can also be put in a simple intuitive form in the case of nonrelativistic drift motion. Since the drift velocity enters the expression for $A_{0}^{\alpha}$ only through relativistic dependence of $\boldsymbol{r}_{\sim}$ on $\boldsymbol{v}_{0} / c$, then, in the case $\boldsymbol{v}_{0} \ll c, \partial A_{0}^{\alpha} / \partial \boldsymbol{v}_{0}$ can be neglected. In this case, the drift canonical momentum is given by $\boldsymbol{P}_{0}=m_{\mathrm{eff}} \boldsymbol{v}_{0}+(e / c) \boldsymbol{A}_{0}$, and the Hamiltonian can be put in the form

$$
H_{0}=\frac{1}{2 m_{\mathrm{eff}}}\left(\boldsymbol{P}_{0}-\frac{e}{c} \boldsymbol{A}_{0}\right)^{2}+m_{\mathrm{eff}} c^{2}+e \phi_{0},
$$

where the effective mass $m_{\mathrm{eff}}$ and the potential energy $\psi_{\text {eff }}=m_{\text {eff }} c^{2}+e \phi_{0}$ may slowly depend on the guiding center location $\boldsymbol{R}_{0}$ and time $t$. Note that even in uniform laser field, $\nabla \psi_{\text {eff }}$ may differ significantly from $e \nabla \phi_{\mathrm{bg}}$ when the amplitude of particle oscillations $r_{\sim}$ exceeds the spatial scale of the background field $l[14,15]$. The regime of slow drift motion superimposed on relativistic oscillations is the one, which is actually realized in many current experiments on intensive laser pulses interaction with rare plasmas. This fact makes the above analysis especially useful from the practical point of view, as it represent a simple tool for studying the actual experimental data. Finally, the well-known nonrelativistic ponderomotive potential [4] can be readily derived from Eq. (22) by keeping the correction to the effective mass, linear with respect to the wave intensity (see also Ref. [12]).

In summary, we showed that oscillating particles immersed into low-frequency background fields behave exactly like drifting particles with effective mass depending on the intensity of the laser field. The intuitive expectation that, by the order of magnitude, the drift motion equations must coincide with those without the laser field if the appropriate relativistic correction of particle mass is introduced, can now be considered proven for various types of background fields. The proposed formulation can be useful for studying a number of plasma phenomena taken place under the action of intense laser radiation.

Authors thank A.A. Balakin for fruitful discussions. The work was supported by the US DOE, under contract DE-AC02-76 CHO3073, and Russian Foundation for Basic Research, grants 01-02-17388, 02-02-06258.
[1] M.D. Perry, D. Pennington, B.C. Stuart et al, Opt. Lett. 24, 160 (1999).

[2] M.H. Key, M.D. Cable, T.E. Cowan et al, Phys. Plasmas 5, 1966 (1998).

[3] L.D. Landau and E.M. Lifshitz, The classical theory of fields (Pergamon Press, Oxford, New York, 1971).

[4] A.V. Gaponov and M.A. Miller, Sov. Phys. JETP 7, 168 (1958).

[5] H. Motz and C.J.H. Watson, Advances in Electronics 23, 153 (1967).

[6] A.G. Litvak, Reviews of Plasma Physics (Energoatomizdat, Moscow, 1980).

[7] V.A. Kozlov, A.G. Litvak and E.V. Suvorov, Zh. Eksp. Teor. Fiz. 26, 148 (1979).

[8] T.W.B. Kibble, Phys. Rev. 150, 1060 (1966).
[9] J.E. Moore and N.J. Fisch, Phys. Plasmas 1, 1105 (1994). [10] J.E. Moore, Phys. Rev. E 59, 2281 (1999).

[11] X. Li, Phys. Rev. E 59, 6048 (1999).

[12] D. Bauer and P. Mulser and W.H. Steeb, Phys. Rev. Lett. 75, 4622 (1995).

[13] M.D. Tokman, Plas. Phys. Rep. 25, 140 (1999).

[14] M.H. Mittleman, Introduction to the Theory of LaserAtom Interactions (Plenum Press, New York, 1993).

[15] M. Pont, N.R. Walet and M. Gavril, Phys. Rev. A 41, 477 (1990).

[16] G.M. Fraiman and A.A. Balakin, Physica D 152, 731 (2001).

[17] J.D. Jackson Classical Electrodynamics (Wiley, New York, 1975). 


\section{External Distribution}

Plasma Research Laboratory, Australian National University, Australia

Professor I.R. Jones, Flinders University, Australia

Professor João Canalle, Instituto de Fisica DEQ/IF - UERJ, Brazil

Mr. Gerson O. Ludwig, Instituto Nacional de Pesquisas, Brazil

Dr. P.H. Sakanaka, Instituto Fisica, Brazil

The Librarian, Culham Laboratory, England

Mrs. S.A. Hutchinson, JET Library, England

Professor M.N. Bussac, Ecole Polytechnique, France

Librarian, Max-Planck-Institut für Plasmaphysik, Germany

Jolan Moldvai, Reports Library, MTA KFKI-ATKI, Hungary

Dr. P. Kaw, Institute for Plasma Research, India

Ms. P.J. Pathak, Librarian, Insitute for Plasma Research, India

Ms. Clelia De Palo, Associazione EURATOM-ENEA, Italy

Dr. G. Grosso, Instituto di Fisica del Plasma, Italy

Librarian, Naka Fusion Research Establishment, JAERI, Japan

Library, Plasma Physics Laboratory, Kyoto University, Japan

Research Information Center, National Institute for Fusion Science, Japan

Dr. O. Mitarai, Kyushu Tokai University, Japan

Library, Academia Sinica, Institute of Plasma Physics, People's Republic of China

Shih-Tung Tsai, Institute of Physics, Chinese Academy of Sciences, People's Republic of China

Dr. S. Mirnov, TRINITI, Troitsk, Russian Federation, Russia

Dr. V.S. Strelkov, Kurchatov Institute, Russian Federation, Russia

Professor Peter Lukac, Katedra Fyziky Plazmy MFF UK, Mlynska dolina F-2, Komenskeho Univerzita, SK-842 15 Bratislava, Slovakia

Dr. G.S. Lee, Korea Basic Science Institute, South Korea

Institute for Plasma Research, University of Maryland, USA

Librarian, Fusion Energy Division, Oak Ridge National Laboratory, USA

Librarian, Institute of Fusion Studies, University of Texas, USA

Librarian, Magnetic Fusion Program, Lawrence Livermore National Laboratory, USA

Library, General Atomics, USA

Plasma Physics Group, Fusion Energy Research Program, University of California at San Diego, USA

Plasma Physics Library, Columbia University, USA

Alkesh Punjabi, Center for Fusion Research and Training, Hampton University, USA

Dr. W.M. Stacey, Fusion Research Center, Georgia Institute of Technology, USA

Dr. John Willis, U.S. Department of Energy, Office of Fusion Energy Sciences, USA

Mr. Paul H. Wright, Indianapolis, Indiana, USA 
The Princeton Plasma Physics Laboratory is operated by Princeton University under contract with the U.S. Department of Energy.

\author{
Information Services \\ Princeton Plasma Physics Laboratory \\ P.O. Box 451 \\ Princeton, NJ 08543
}

Phone: 609-243-2750

Fax: 609-243-2751

e-mail: pppl_info@pppl.gov

Internet Address: http://www.pppl.gov 